\title{
Hommage à John Walton (Lord Walton of Detchant), 1922-2016
}

Michel Fardeau

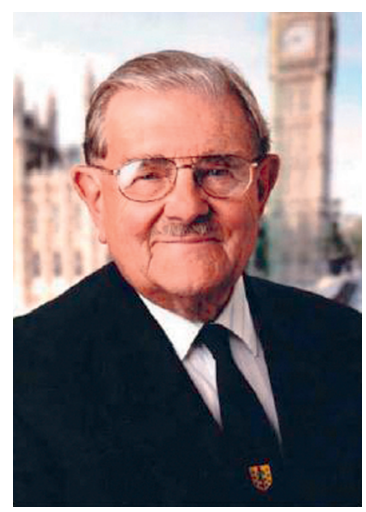

Le Professeur John Walton - Lord Walton of Detchant - est décédé le 21 avril dernier. Avec lui disparaît le fondateur de la myologie en Europe, et l'une des personnalités "premières" de la myologie dans le monde.

John Walton était né en 1922 dans un petit village minier du nord-est de l'Angleterre. Il restera toujours très attaché à sa région natale. À 18 ans, en 1940, demandant à s'engager dans la Royal Air Force, il n'y sera pas admis, l'Angleterre ayant décidé de former en priorité de nouveaux médecins. Il fera donc des études de médecine "accélérées " à Newcastle-upon-Tyne. À la fin de ses études, il sera appelé à servir dans la "Navy" comme Commandant en second d'un navire-hôpital, et servira en particulier au Moyen Orient. De retour à la vie civile, en 1948, il sera orienté par deux de ses "mentors", le Professeur Nattrass, de Durham, et le Docteur Henry Miller, de Newcastle, vers la neurologie. C'est alors qu'il examinera tous les patients identifiés comme "dystrophie musculaire " dans le Comté du Northumberland, et qu'il en tirera une classification enfin claire de ces maladies, qui sera publiée, avec Fred Nattrass, en 1954, dans Brain. Clarté d'esprit, sens de l'organisation, rapidité de décision auront été parmi les plus éminentes qualités de "John": c'est ainsi qu'il aimait qu'on l'appelât.

Décidé à continuer à travailler dans le domaine des maladies neuromusculaires, il obtint un " $\mathrm{fel}$ lowship" de la Nuffield Foundation, pour aller passer une année à Boston, au " Mass General Hospital " auprès de Ray Adams. Il y approfondit ses connaissances cliniques et histopathologiques des affections neuromusculaires. Après avoir passé une année supplémentaire de formation auprès du Docteur Carmichael, à Queen Square (Londres), il revint à Newcastle, avec un "grant" de la "Muscular Dystrophy Association of America", qui lui permit de recruter ses premiers collaborateurs en clinique, électromyographie et biochimie. Nommé
Consultant en 1958, il obtint de pouvoir monter un premier laboratoire de recherche en myopathologie. L'attribution de nouveaux " grants " lui permit d'étoffer son équipe, et des "fellows " commencèrent à venir du monde entier: le premier centre européen de myologie était ainsi créé.

Ce n'est que plus tard, en 1983, qu'il quittera Newcastle pour Oxford, où il sera nommé au poste prestigieux de "Warden of Green College" avant de revenir, en 1989, à Newcastle. Entre temps, il aura été anobli par la Reine, et de "Sir John " il sera devenu "Lord Walton of Detchant ". Ses responsabilités dans le développement des différentes institutions britanniques et mondiales seront vite impressionnantes.

John aura eu une influence décisive dans le développement de la myologie dans nombre de pays dans le monde. Pour la France, il eut un rôle majeur dans le développement du laboratoire de la Salpêtrière. Ami, à travers Henri Miller et Ray Adams, du Professeur Raymond Garcin, il joua un rôle décisif lorsque ce laboratoire fut fragilisé par la disparition rapide de monsieur Garcin en 1971. Il vint alors lui-même de Newcastle plaider auprès du Doyen de la Pitié-Salpêtrière, le Professeur Paul Castaigne, pour le maintien de ce laboratoire : ce qui fut alors acquis. Dans ces années, les échanges et visites se multiplièrent entre Paris et Newcastle. John, et son épouse Lady Betty, aimaient beaucoup notre pays, dont ils pratiquaient "gentiment" la langue, y compris en chansons, surtout à la fin d'un bon repas. John aura été un garant très sûr du développement de la myologie dans notre pays. Sa disparition est pleurée par tous ses collaborateurs et amis, de Newcastle et du monde entier. Notre peine est égale à la leur, à la hauteur de l'admiration que nous avions pour cet homme et de ce que nous lui devons.

A Tribute to John Walton (Lord Walton of Detchant) $1922-2016$

\section{LIENS D'INTÉRÊT}

L'auteur déclare n'avoir aucun lien d'intérêt concernant les données publiées dans cet article.

\section{NOTE BIBLIOGRAPHIQUE}

John Walton a conté toute son histoire personnelle dans un livre "The Spice of Life: from Northumberland to World Neurology ", London, Royal Society of Medicine, 1993. 